OPEN ACCESS

Edited by:

Paola Patrignani,

University of Studies G. d'Annunzio

Chieti and Pescara, Italy

Reviewed by:

Francesco Taus,

University of Verona, Italy

Francesco Sessa,

University of Foggia, Italy

*Correspondence:

Liang Guo

guoliangwhzn@163.com

Xinghuan Wang

wangxinghuan@whu.edu.cn

Xiaohui Wu

wuxiaohui1971@sina.com

${ }^{\dagger}$ These authors have contributed equally to this work

Specialty section:

This article was submitted to

Pharmacogenetics and Pharmacogenomics,

a section of the journal

Frontiers in Pharmacology

Received: 29 May 2020

Accepted: 06 July 2020

Published: 17 July 2020

Citation:

Liu Z, Li J, Chen D, Gao R, Zeng W, Chen S, Huang Y, Huang J, Long W, Li M, Guo L, Wang X and Wu X (2020) Dynamic Interleukin-6 Level Changes as a Prognostic Indicator in Patients With COVID-19.

Front. Pharmacol. 11:1093. doi: 10.3389/fphar.2020.01093

\section{Dynamic Interleukin-6 Level Changes as a Prognostic Indicator in Patients With COVID-19}

\author{
Zeming $\mathrm{Liu}^{1+}$, Jinpeng $\mathrm{Li}^{2 \dagger}$, Danyang Chen ${ }^{1+}$, Rongfen $\mathrm{Gao}^{3 \dagger}$, Wen Zeng ${ }^{4 \dagger}$, \\ Sichao Chen ${ }^{1}$, Yihui Huang ${ }^{1}$, Jianglong Huang ${ }^{1}$, Wei Long ${ }^{1}$, Man $\mathrm{Li}^{1}{ }^{1}$, Liang Guo ${ }^{1 *}$, \\ Xinghuan Wang ${ }^{5 *}$ and Xiaohui Wu ${ }^{6 *}$
}

\begin{abstract}
${ }^{1}$ Department of Plastic Surgery, Zhongnan Hospital of Wuhan University, Wuhan, China, ${ }^{2}$ Department of Thyroid and Breast Surgery, Zhongnan Hospital of Wuhan University, Wuhan, China, ${ }^{3}$ Department of Rheumatology and Immunology, Tongji Hospital, Tongji Medical College, Huazhong University of Science and Technology, Wuhan, China, ${ }^{4}$ Department of Ophthalmology, Zhongnan Hospital of Wuhan University, Wuhan, China, ${ }^{5}$ Department of Urology, Zhongnan Hospital of Wuhan University, Wuhan, China, ${ }^{6}$ Department of Neurosurgery, Zhongnan Hospital of Wuhan University, Wuhan, China
\end{abstract}

Background: Interleukin-6 (IL-6), a proinflammatory cytokine, has been reported to be associated with disease severity and mortality in patients with coronavirus disease 2019 (COVID-19). Yet, dynamic changes in IL-6 levels and their prognostic value as an indicator of lung injury in COVID-19 patients have not been fully elucidated.

Objective: To validate whether IL-6 levels are associated with disease severity and mortality and to investigate whether dynamic changes in IL-6 levels might be a predictive factor for lung injury in COVID-19 patients.

Methods: This retrospective, single-center study included 728 adult COVID-19 patients and used data extracted from electronic medical records for analyses.

Results: The mortality rate was higher in the elevated IL-6 group than in the normal IL-6 group (0.16 vs 5\%). Cox proportional hazards and logistic regression analyses for survival (adjusted hazard ratio, 10.39; 95\% confidence interval [Cl], 1.09-99.23; $p=0.042$ ) and disease severity (adjusted odds ratio, 3.56; 95\% Cl, 2.06-6.19; $p<0.001$ ) revealed similar trends. Curve-fitting analyses indicated that patient computed tomography (CT) scores peaked on days 22 and 24. An initial decline in IL-6 levels on day 16 was followed by resurgence to a peak, nearly in tandem with the CT scores.

Conclusion: Increased IL-6 level may be an independent risk factor for disease severity and in-hospital mortality and dynamic IL-6 changes may serve as a potential predictor for lung injury in Chinese COVID-19 patients. These findings may guide future treatment of COVID-19 patients.

Keywords: COVID-19, interleukin-6, SARS-CoV-2, CT scores, infectious disease, lung injury 


\section{INTRODUCTION}

The outbreak of coronavirus disease (COVID-19), first detected in Wuhan, China, in December 2019, has been categorized as a pandemic since March 2020. As COVID-19 continues to spread worldwide, 5,722,859 cases and 356,279 deaths have been reported as of May 28, 2020.

The Leishenshan (Thunder God Mountain) Hospital, the largest makeshift hospital in China, played an essential role in the battle against COVID-19 in Wuhan, China. The hospital had the capacity to accommodate 1,600 patients and was well equipped with intensive care units and operating rooms as well as clinical laboratories providing relatively standardized and systematic medical information on COVID-19 patients.

Interleukin 6 (IL-6) and other components of the inflammatory cascade contribute to host defense against infections. However, excessive synthesis of IL-6 can lead to a severe acute systemic inflammatory response known as a "cytokine storm," which confers increased risks of vascular hyperpermeability, multiorgan failure, and eventually death (Meduri et al., 1995). This pathophysiological process, which is supported by many studies, suggests that higher IL-6 levels may be an important predictor of COVID-19 severity (Cai et al., 2020; Chen et al., 2020). However, the role of IL-6 as an independent predictor of severity and mortality in hospitalized COVID-19 patients has not been validated.

Acute respiratory distress syndrome (ARDS), one of the major factors contributing to mortality in COVID-19 patients, is similar to severe community-acquired pneumonia caused by other viruses or bacteria (Ranieri et al., 2012; Jose and Manuel, 2020). The pathophysiological process of ARDS involves a cytokine storm caused by the overproduction of early response proinflammatory cytokines. Theoretically, monitoring the IL-6 level is crucial to understanding the pathophysiological process of ARDS; however, whether IL-6 levels can predict the severity of pulmonary injury has not been fully investigated in patients with COVID-19.

Thus, in this study, we aimed to determine whether IL-6 levels are associated with disease severity and mortality in COVID-19 patients and investigate whether dynamic changes in IL-6 levels are predictive of pulmonary lesions caused by ARDS in hospitalized COVID-19 patients.

\section{METHODS}

\section{Patient Selection}

This retrospective study included 1,880 confirmed COVID-19 patients who were admitted to the Leishenshan Hospital in

\footnotetext{
Abbreviations: ARDS, acute respiratory distress syndrome; AUC, area under the curve; CI, confidence interval; COVID-19, coronavirus disease 2019; CT, computed tomography; GGO, ground-glass opacity; HR, hazard ratio; Ig, immunoglobulin; IL-6, interleukin-6; INR, international normalized ratio; MERS-CoV, Middle East respiratory syndrome coronavirus; OR, odds ratio; ROC, receiver-operating characteristic; SARS, severe acute respiratory syndrome coronavirus; SARS-CoV-2, severe acute respiratory syndrome coronavirus 2 .
}

Wuhan, China, between February 9 and April 9, 2020. Exclusion criteria were patients: younger than 18, missing data on IL-6 levels, acute myocardial infarction during hospitalization, acute pancreatitis, or transferred to another hospital. The dataset included in the final analysis consisted of 728 cases (Figure S1).

\section{Data Collection and Primary Outcomes}

Detailed patient information, including demographic characteristics, history of comorbidities, symptoms, laboratory findings, computed tomography (CT) findings, length of hospital stay, oxygen support, illness severity on admission, and treatment, was collected from the electronic patient records stored in the hospital information management system and entered into a customized Excel form (Microsoft, Redmond, WA, USA).

The primary outcomes were patient survival and COVID-19 severity during hospitalization. Based on the $7^{\text {th }}$ Interim Guidance for the Diagnosis and Treatment of COVID-19 published by the National Health Commission of China, each patient was classified as mild, common, severe, or critical.

\section{Evaluation of CT Images}

As chest CT has a high sensitivity for the diagnosis of COVID19, studies have advocated for the use of CT images to evaluate the severity of acute respiratory syndrome (Wong et al., 2004; Ai et al., 2020; Lyu et al., 2020). Furthermore, radiographic scores have been shown to play an important role in the diagnosis of severe acute respiratory syndrome (SARS) between November 2002 and August 2003 (Wong et al., 2004). In our study, chest CT images were independently inspected by two radiologists who were blinded to the patients' clinical data. An semiquantitative scoring system, based on previous studies and the characteristics of COVID-19, was adopted for the assessment of pulmonary inflammation. Score 1 was generated based on CT imaging features such as ground-glass opacities (GGOs), reticulate or cord-like changes, consolidation, and pleural effusion. Each imaging feature received 1 point. While Score 1 comprised the sum of the points, Score 2 was generated based on the area of lung lobe involvement and graded from 0 to 4 as follows: no involvement, 0 ; $<25 \%$ involvement, $1 ; 26-50 \%$ involvement, 2 ; $51-75 \%$ involvement, 3 ; and $76-100 \%$ involvement, 4 . The overall score was the sum of Scores 1 and 2.

\section{Statistical Analyses}

Patients were assigned to either the elevated or normal IL-6 group based on an IL- 6 cutoff value of $7 \mathrm{pg} / \mathrm{ml}$ (obtained from medical records). Continuous variables are presented as medians and interquartile ranges (IQRs), whereas categorical variables are expressed as the number of patients (-percentages).

The Kruskal-Wallis or Mann-Whitney $U$ test was used for intergroup comparisons between the elevated IL-6 and normal IL-6 groups with regard to the means of continuous variables. For categorical variables, intergroup comparisons were performed using the chi-squared test or Fisher's exact test.

Cox proportional hazards regression models were used to determine the effect of IL-6 levels on overall survival. We used a multivariate Cox proportional hazards regression model 
adjusted for variables such as age, history of cardiovascular disease, lymphocyte count, D-dimer level, and lactate dehydrogenase level. Survival analysis was conducted using Kaplan-Meier survival curves and the log-rank test or MantelHaenszel test. Moreover, the cumulative hazard function for COVID-19 progression in both groups was analyzed. The relationship between IL-6 levels and CT scores of lung injury was assessed using a curve-fitting analysis. Receiver-operating characteristic (ROC) curve analysis was used to evaluate the predictive ability for death and disease severity.

All statistical analyses were performed using $\mathrm{R}$ version 3.6.1 (https://www.r-project.org/, R Core Team, Vienna, Austria) and EmpowerStats version 2.0- (http://www.empowerstats.com/cn/, $\mathrm{X} \& \mathrm{Y}$ Solutions, Inc., Boston, MA). A two-sided $p$-value $<0.05$ was considered statistically significant.

\section{Ethics Approval and Informed Consent}

The study protocol was approved by the Research Ethics Committee of the Zhongnan Hospital of Wuhan University (approval no.: 2020074). The Research Ethics Committee waived the need for informed consent from the patients due to the urgent need for research insights in the context of this rapidly evolving infectious disease. All procedures undertaken in this study were in accordance with the ethical standards of the institutional and national research committee as well as the 1964 Declaration of Helsinki and its later amendments and other comparable ethical standards.

\section{RESULTS}

\section{Baseline Characteristics of the Study Participants}

The baseline characteristics of 728 patients are listed in Table $\mathbf{1}$. The median ages in the normal and elevated IL- 6 groups were 57 and 68 years, respectively. A total of seven (0.96\%) patients died. In the normal IL- 6 group, the majority of patients (54.4\%) were female. In contrast, there was a higher proportion (54.2\%) of male patients in the elevated IL- 6 group. Cardiovascular diseases were more prevalent in the elevated IL- 6 group $(44.17 \%)$ than in the normal IL-6 group (20.23\%); similarly, neurological diseases were also more common in the elevated IL- 6 than in the normal IL-6 group (11.76 vs. 4.93\%). As shown in Table 2, the levels of routine blood test parameters, including leukocyte count, neutrophil count, were significantly higher in the elevated IL-6 group than in the normal IL-6 group. Moreover, the lymphocyte, erythrocyte, and platelet counts and hemoglobin concentration were reduced in the elevated IL-6 group. With regard to liver function parameters, the direct bilirubin level was higher and accompanied by notable reductions in albumin and total protein levels in the elevated IL-6 group. An evaluation of renal function showed significantly elevated serum creatinine, urea, and cystatin $C$ levels. Lactate dehydrogenase levels were increased in the elevated IL-6 group. Significant abnormalities in blood coagulation parameters, such as prolonged prothrombin time and activated partial thromboplastin time, higher International Normalized Ratio, and increased D-dimer levels, were noted in the elevated IL-6 group. Moreover, the proportion (84.62\%) of patients with positivity for immunoglobulin $\mathrm{G}$ ( IgG) was lower in the elevated IL- 6 group than in the normal IL- 6 group $(94.03 \%)$. The clinical treatment and outcome analyses revealed that more patients in the elevated IL- 6 group were likely to require antibiotics, antiviral drugs, anticoagulants, corticosteroids, and vitamin $\mathrm{C}$ therapy. Furthermore, in the normal IL-6 group, there was a higher use of antimalarial drugs $(83.93 \%)$ and traditional Chinese medicine $(88.82 \%)$. Patients in the elevated IL-6 group were more likely to be admitted to the intensive care unit during hospitalization $(20.00 \%)$ than patients in the normal IL-6 group $(0.82 \%)$ (Table 3).

TABLE 1 | Demographic characteristics and symptoms of 728 patients with COVID-19.

\begin{tabular}{|c|c|c|c|c|c|}
\hline Covariates & Levels & Normal IL-6(n = 608), n (\%) & Elevated IL-6(n = 120), $n(\%)$ & All patients $(n=728), n(\%)$ & $P$ value \\
\hline & male & $277(45.6 \%)$ & $65(54.2 \%)$ & $342(46.98)$ & \\
\hline \multirow{5}{*}{ Any comorbidity } & & 171 (60.21\%) & 76 (74.51\%) & 247 (63.99\%) & 0.010 \\
\hline & Cardiovascular diseases & $123(20.23 \%)$ & $53(44.17 \%)$ & $176(24.18 \%)$ & $<0.001$ \\
\hline & Malignancy & $19(6.69 \%)$ & $10(9.80 \%)$ & $29(7.5 \%)$ & 0.306 \\
\hline & Digest system diseases & $12(4.23 \%)$ & $9(8.82 \%)$ & $21(5.4 \%)$ & 0.079 \\
\hline & Neurological diseases & $14(4.93 \%)$ & $12(11.76 \%)$ & $26(6.7 \%)$ & 0.018 \\
\hline \multicolumn{6}{|l|}{ Initial symptoms } \\
\hline & Fever or fatigue & $213(80.08 \%)$ & 75 (76.53\%) & $288(79.12 \%)$ & 0.461 \\
\hline & Respiratory symptoms & $217(81.58 \%)$ & $82(83.67 \%)$ & $299(82.14 \%)$ & 0.644 \\
\hline
\end{tabular}


TABLE 2 | Serum biochemical parameters and blood coagulation test results of 728 patients with COVID-19.

\section{Covariate}

Median (IQR)/n (\%)

\section{Liver function parameters}

Alanine aminotransferase, U/L

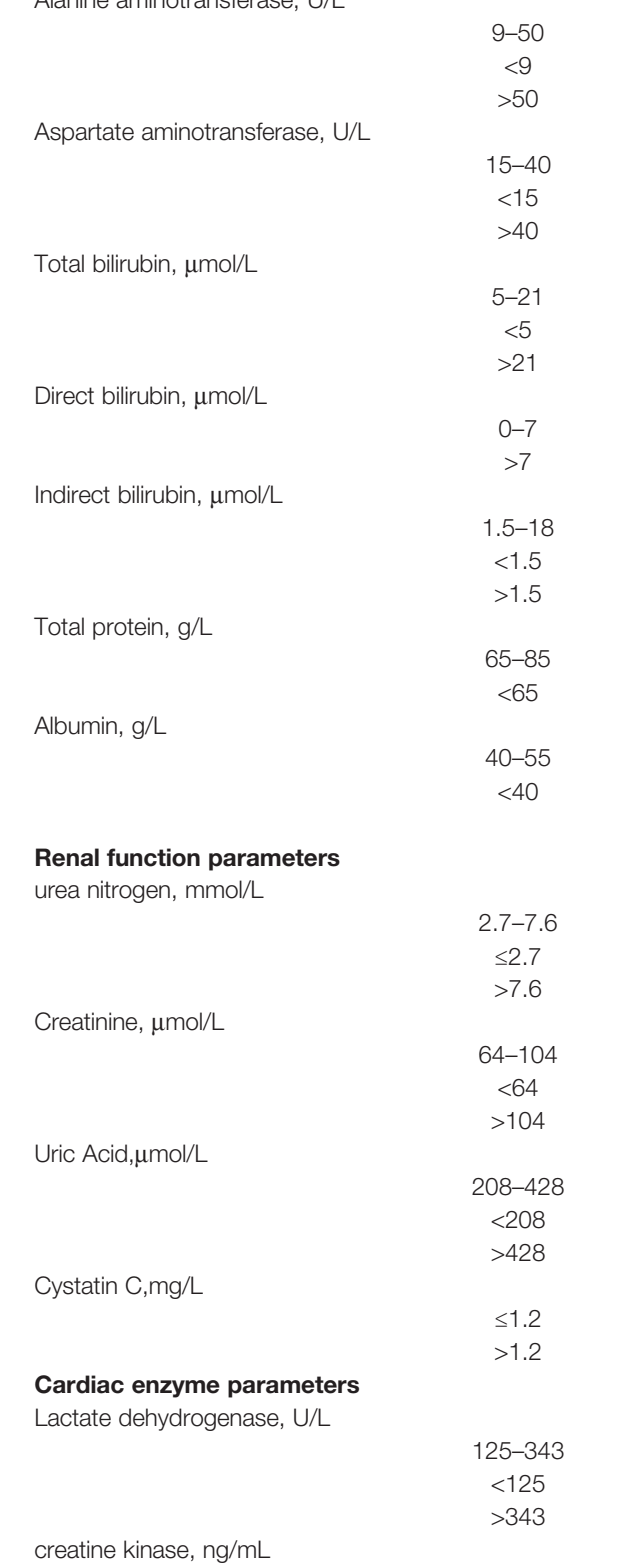

Normal
male $>171$, female $>145$

Coagulation function parameters

Prothrombin time, s

$$
\begin{gathered}
9.4-12.5 \\
<9.4 \\
>12.5
\end{gathered}
$$

Activated partial thromboplastin time, s

$$
\begin{gathered}
25.1-36.5 \\
<25.1 \\
>36.5
\end{gathered}
$$

Thrombin time, s

$26.00(16.00-42.00)$
$471(77.72 \%)$
$24(3.96 \%)$
$111(18.32 \%)$
$21.00(16.00-28.00)$
$455(75.08 \%)$
$97(16.01 \%)$
$54(8.91 \%)$
$9.10(7.20-11.97)$
$557(91.91 \%)$
$34(5.61 \%)$
$15(2.48 \%)$
$3.05(2.40-4.00)$
$584(96.37 \%)$
$22(3.63 \%)$
$5.70(4.40-8.00)$
$504(98.63 \%)$
$2(0.39 \%)$
$5(0.98 \%)$

$66.50(62.80-69.90)$ $375(61.88 \%)$ $231(38.12 \%)$ $38.0(35.7-40.5)$ $184(30.36 \%)$ $422(69.64 \%)$

\section{$4.70(3.80-5.70)$ $554(91.57 \%)$ $27(4.46 \%)$ 24 (3.97\%) $63.3(53.7-74.7)$ 271 (44.79\%) 314 (51.90\%) 20 (3.31\%)}

303.00 (245.00-367.00) 506 (83.64\%) $26(4.30 \%)$ $73(12.07 \%)$ $0.91(0.80-1.01)$ $559(92.40 \%)$ $46(7.60 \%)$

184.00 (161.00-213.00) $546(94.30 \%)$

$17(2.94 \%)$

$16(2.76 \%)$

$53.0(37.0-77.0)$

$$
551(95.16 \%)
$$

28 (4.84\%) $546(95.62 \%)$ 0

25 (4.38\%)

$27.0(24.3-30.3)$

$360(63.05 \%)$

188 (32.92\%)

$23(4.03 \%)$

$17.6(17.0-18.4)$

\section{$11.3(10.9-11.6)$}

\section{Normal IL-6 (n = 608)
Median (IQR)/n (\%) Median (IQR)/n (\%)}

23.00 (13.00-38.50) $89(74.79 \%)$ $10(8.40 \%)$ $20(16.81 \%)$

22.00 (16.00-34.00)

$71(59.66 \%)$

$22(18.49 \%)$

$26(21.85 \%)$

$9.10(7.20-11.97)$ $100(84.03 \%)$

$7(5.88 \%)$

$12(10.08 \%)$

$4.00(2.80-5.95)$

$97(81.51 \%)$

$22(18.49 \%)$

5.05 (4.03-7.07)

$99(97.06 \%)$

$0(0.00 \%)$

3 (2.94\%)

$62.70(58.45-66.45)$

40 (33.61\%)

79 (66.39\%)

$34.2(31.4-37.5)$

$9(7.56 \%)$

$110(92.44 \%)$

\section{$5.20(4.00-6.95)$ \\ $85(71.43 \%)$ \\ $10(8.40 \%)$ \\ $24(20.17 \%)$ \\ $67.1(54.2-84.6)$ \\ 46 (38.66\%) \\ 53 (44.54\%) \\ $20(16.81 \%)$}

\subsection{0 (199.00-357.50)}

$85(71.43 \%)$

$20(16.81 \%)$

$14(11.76 \%)$

$1.07(0.92-1.36)$

$79(66.39 \%)$

$40(33.61 \%)$

$25.15(184.00-284.75)$
$97(85.09 \%)$
$1(0.88 \%)$
$16(14.04 \%)$
$44.4(26.2-61.0)$

\section{$106(92.98 \%)$}

$8(7.02 \%)$

\section{$11.8(11.3-12.5)$}

$90(76.92 \%)$

0

$27(23.08 \%)$

$29.2(26.8-33.0)$

$86(73.50 \%)$

$15(12.82 \%)$

$16(13.68 \%)$

$17.0(16.1-17.9)$
All patients $(\mathrm{n}=\mathbf{7 2 8}) \quad \mathrm{P}$ value Reference range Median (IQR)/n (\%)

$\begin{array}{ccc}25.00(16.00-42.00) & 0.177 & 9-50 \\ 560(77.24 \%) & 0.11 & \\ 34(4.69 \%) & & \\ 131(18.07 \%) & & \\ 21.00(16.00-29.00) & 0.062 & 15-40 \\ 526(72.55 \%) & <0.001 & \\ 119(16.41 \%) & & \\ 80(11.03 \%) & & \\ 9.20(7.10-12.20) & 0.16 & 5.0-21.0 \\ 657(90.62 \%) & <0.001 & \\ 41(5.66 \%) & & \\ 27(3.72 \%) & & \\ 3.10(2.40-4.30) & <0.001 & 0-7 \\ 681(93.93 \%) & <0.001 & \\ 44(6.07 \%) & & \\ 5.60(4.30-7.80) & 0.049 & 1.5-18 \\ 603(98.37 \%) & 0.231 & \\ 2(0.33 \%) & & \\ 8(1.31 \%) & & \end{array}$

$65.90(61.90-69.70) \quad<0.001$

65-85 $415(57.24 \%) \quad<0.001$ $310(42.76 \%)$

$37.70(35.00-40.20) \quad<0.001$ $193(26.62 \%) \quad<0.001$ $532(73.38 \%)$

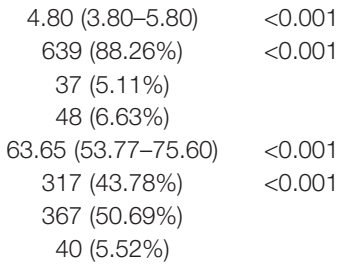

$27.50(24.70-30.50) \quad<0.001 \quad 25.1-36.5$
$10.3-16.6$

\section{$2.7-7.6$}

64.0-104.0

$208-428$

125-343

female $\leq 145$

$9.4-12.5$

(1)

(Continued) 
TABLE 2 | Continued

\begin{tabular}{|c|c|c|c|c|c|c|}
\hline Covariate & & $\begin{array}{c}\text { Normal IL-6 (n = 608) } \\
\text { Median (IQR)/n (\%) }\end{array}$ & $\begin{array}{c}\text { Elevated IL-6 }(\mathbf{n}=120) \\
\text { Median (IQR)/n (\%) }\end{array}$ & $\begin{array}{l}\text { All patients }(\mathrm{n}=728) \\
\text { Median (IQR)/n (\%) }\end{array}$ & $P$ value & Reference range \\
\hline & $10.3-16.6$ & $81(14.19 \%)$ & $42(35.90 \%)$ & $123(17.88 \%)$ & $<0.001$ & \\
\hline & $>16.6$ & 490 (85.81\%) & 75 (64.10\%) & 565 (82.12\%) & & \\
\hline \multirow[t]{7}{*}{ International Normalized Ratio } & & $0.97(0.93-1.00)$ & $1.02(0.97-1.08)$ & $0.97(0.93-1.01)$ & $<0.001$ & $0.85-1.15$ \\
\hline & $0.85-1.15$ & 566 (99.12\%) & 109 (93.16\%) & 675 (98.11\%) & $<0.001$ & \\
\hline & $>1.15$ & 5 (0.88\%) & 8 (6.84\%) & 13 (1.89\%) & & \\
\hline & & $2.90(2.47-3.66)$ & $3.73(3.00-4.63)$ & $3.00(2.51-3.82)$ & $<0.001$ & $2.38-4.98$ \\
\hline & $2.38-4.98$ & 381 (66.73\%) & 55 (47.01\%) & 436 (63.37\%) & $<0.001$ & \\
\hline & $<2.38$ & 111 (19.44\%) & 13 (11.11\%) & 124 (18.02\%) & & \\
\hline & $>4.98$ & 79 (13.84\%) & $49(41.88 \%)$ & $128(18.60 \%)$ & & \\
\hline \multirow[t]{3}{*}{ D-dimer, g/L } & & $0.37(0.21-0.82)$ & $1.03(0.56-2.61)$ & $0.44(0.23-1.04)$ & $<0.001$ & $0-0.50$ \\
\hline & $0-0.5$ & 348 (60.95\%) & 28 (23.93\%) & 376 (54.65\%) & $<0.001$ & \\
\hline & $>0.5$ & $223(39.05 \%)$ & $89(76.07 \%)$ & $312(45.35 \%)$ & & \\
\hline \multicolumn{7}{|l|}{ Routine blood tests } \\
\hline \multirow[t]{4}{*}{ Leucocyte count, $\times 10^{9} / \mathrm{L}$} & WBC & $5.66(4.65-6.71)$ & $6.46(4.84-7.80)$ & $5.7(4.7-6.9)$ & $<0.001$ & $3.5-9.5$ \\
\hline & $3.5-9.5$ & 550 (90.61\%) & 92 (76.67\%) & 642 (88.31\%) & $<0.001$ & \\
\hline & $<3.5$ & $40(6.59 \%)$ & 10 (8.33\%) & 50 (6.88\%) & & \\
\hline & $>9.5$ & 17 (2.80\%) & 18 (15.00\%) & 35 (4.81\%) & & \\
\hline \multirow[t]{4}{*}{ Neutrophil count, $\times 10^{9} / \mathrm{L}$} & & 3.18 (2.48-4.05) & $4.23(3.14-5.86)$ & $3.29(0.06-33.01)$ & $<0.001$ & $1.8-6.3$ \\
\hline & $1.8-6.3$ & 533 (87.81\%) & $87(72.50 \%)$ & 620 (85.28\%) & $<0.001$ & \\
\hline & $<1.8$ & $48(7.91 \%)$ & $7(5.83 \%)$ & $55(7.57 \%)$ & & \\
\hline & $>6.3$ & 26 (4.28\%) & 26 (21.67\%) & $52(7.15 \%)$ & & \\
\hline \multirow[t]{4}{*}{ Lymphocyte count, $\times 10^{9} / \mathrm{L}$} & & 1.64 (1.33-1.98) & $1.14(0.81-1.52)$ & $1.58(0.17-3.56)$ & $<0.001$ & $1.1-3.2$ \\
\hline & $1.1-3.2$ & 375 (61.78\%) & 44 (36.67\%) & 419 (57.63\%) & $<0.001$ & \\
\hline & $<1.1$ & 39 (6.43\%) & 25 (20.83\%) & $64(8.80 \%)$ & & \\
\hline & $>3.2$ & 193 (31.80\%) & 51 (42.50\%) & 244 (33.56\%) & & \\
\hline \multirow[t]{4}{*}{ Erythrocyte count, $\times 10^{12} / \mathrm{L}$} & & $4.13(3.82-4.46)$ & $3.66(3.18-4.19)$ & $4.08(1.78-6.19)$ & $<0.001$ & $4.3-5.8$ \\
\hline & $4.3-5.8$ & 207 (34.10\%) & $21(17.50 \%)$ & 228(31.36\%) & $<0.001$ & \\
\hline & $<4.3$ & 397 (65.40\%) & 96 (80.00\%) & $493(67.81 \%)$ & & \\
\hline & $>5.8$ & $3(0.49 \%)$ & $3(2.50 \%)$ & $6(0.83 \%)$ & & \\
\hline \multirow[t]{4}{*}{ Monocyte count, $\times 10^{9} / \mathrm{L}$} & & $0.50(0.40-0.62)$ & $0.56(0.41-0.73)$ & $0.50(0.03-2.20)$ & 0.032 & $0.1-0.6$ \\
\hline & $0.1-0.6$ & 115 (18.95\%) & 34 (28.33\%) & 149 (20.50\%) & 0.005 & \\
\hline & $<0.1$ & $0(0.00 \%)$ & $1(0.83 \%)$ & $1(0.14 \%)$ & & \\
\hline & $>0.6$ & 492 (81.05\%) & 85 (70.83\%) & 577 (79.37\%) & & \\
\hline \multirow[t]{4}{*}{ Hemoglobin, g/L } & & $126.00(116.00-137.00)$ & 111.50 (98.75-122.25) & $124.00(50.00-178.00)$ & $<0.001$ & $130.0-175.0$ \\
\hline & $130.0-175.0$ & 231 (38.06\%) & $21(17.50 \%)$ & 252 (34.66\%) & $<0.001$ & \\
\hline & $<130.0$ & 376 (61.94\%) & 97 (80.83\%) & $473(65.06 \%)$ & & \\
\hline & $>175.0$ & $0(0.00 \%)$ & $2(1.67 \%)$ & $2(0.28 \%)$ & & \\
\hline \multirow[t]{4}{*}{ Platelet count, $\times 10^{9} / \mathrm{L}$} & & 231.00 (192.00-277.00) & 205.00 (151.00-270.75) & 229.00 (187.00-277.00) & 0.002 & 125.0-350.0 \\
\hline & 125.0-350.0 & $541(89.13 \%)$ & $90(75.00 \%)$ & $631(86.80 \%)$ & $<0.001$ & \\
\hline & $<125.0$ & $18(2.97 \%)$ & $16(13.33 \%)$ & $36(4.95 \%)$ & & \\
\hline & $>350.0$ & $48(7.91 \%)$ & $14(11.67 \%)$ & $60(8.25 \%)$ & & \\
\hline \multicolumn{7}{|l|}{ Other serum tests } \\
\hline \multirow[t]{3}{*}{ Procalcitonin, ng/ml } & & $0.04(0.02-0.05)$ & 0.07 (0.05-0.13) & $0.04(0.03-0.06)$ & $<0.001$ & $<0.05$ \\
\hline & $<0.05$ & 401 (71.4\%) & 27 (23.7\%) & 428 (63.31\%) & $<0.001$ & \\
\hline & $>=0.05$ & $161(28.6 \%)$ & $87(76.3 \%)$ & 248 (36.69\%) & & \\
\hline \multirow[t]{3}{*}{ SARS-CoV-2 IgM } & & & & & 0.905 & \\
\hline & No & 190 (65.97\%) & 45 (65.22\%) & 235 (65.83\%) & & \\
\hline & Yes & 98 (34.03\%) & $24(34.78 \%)$ & $122(34.17 \%)$ & & \\
\hline \multicolumn{7}{|l|}{ SARS-CoV-2 lgG } \\
\hline & No & $16(5.97 \%)$ & $10(15.38 \%)$ & $26(7.81 \%)$ & 0.011 & \\
\hline & Yes & 252 (94.03\%) & 55 (84.62\%) & 307 (92.19\%) & & \\
\hline
\end{tabular}

\section{Univariate and Multivariate Analyses in Patients With Elevated IL-6 Levels}

As shown in Table 4, we conducted a univariate analysis to identify whether an elevated IL-6 level was significantly associated with mortality (hazard ratio [HR], 30.54; 95\% confidence interval [CI], 3.68-253.74; $p<0.002$ ). In the next-step multivariate Cox regression analysis, IL-6 was identified as a significant indicator of mortality (HR, 10.39; 95\% CI, 1.09-99.23; $p=0.042$ ). Furthermore, the ordinal logistic regression model indicated that IL-6 was associated with disease severity in both the univariate (OR, 9.33; 95\% CI, 5.72-15.22; $p<0.001)$ and multivariate (OR, 3.56; 95\% CI, 2.06-6.19; $p<0.001$ ) analyses (Table 5). 
TABLE 3 | Clinical treatments and outcomes of 728 patients with COVID-19.

\begin{tabular}{|c|c|c|c|c|c|}
\hline Covariates & Levels & $\begin{array}{c}\text { Normal IL-6 } \\
\text { (n=608) n (\%) }\end{array}$ & $\begin{array}{c}\text { Elevated IL-6 } \\
(n=120) \text { n (\%) }\end{array}$ & $\begin{array}{l}\text { All patients } \\
\begin{array}{c}(\mathrm{n}=728) \\
n(\%)\end{array}\end{array}$ & $P$ value \\
\hline \multicolumn{6}{|l|}{ Drugs } \\
\hline & Antibiotic & $149(24.51 \%)$ & $66(55.00 \%)$ & $215(29.53 \%)$ & $<0.001$ \\
\hline & Antiviral drugs & $271(44.57 \%)$ & $75(62.50 \%)$ & $346(47.53 \%)$ & $<0.001$ \\
\hline & Antimalarial drugs & $47(83.93 \%)$ & $5(16.67 \%)$ & $52(60.47 \%)$ & $<0.001$ \\
\hline & Anticoagulants & $30(4.93 \%)$ & $46(38.33 \%)$ & 76 (10.44\%) & $<0.001$ \\
\hline & Corticosteroid & $27(4.44 \%)$ & $23(19.17 \%)$ & $50(6.87 \%)$ & $<0.001$ \\
\hline & Vitamin C & $112(18.42 \%)$ & $36(30.00 \%)$ & $148(20.33 \%)$ & 0.004 \\
\hline & Traditional Chinese medicine & $540(88.82 \%)$ & $98(81.67 \%)$ & $638(87.64 \%)$ & 0.03 \\
\hline \multicolumn{6}{|l|}{ Oxygen support } \\
\hline & Low-flow nasal cannula & 76 (85.39\%) & $21(60.00 \%)$ & 97 (78.23\%) & 0.002 \\
\hline & Non-invasive ventilation or high-flow nasal cannula & $13(14.61 \%)$ & $10(28.57 \%)$ & $23(18.55 \%)$ & $<0.001$ \\
\hline & Invasive mechanical ventilation & $0(0.00 \%)$ & $3(8.57 \%)$ & $3(2.42 \%)$ & $<0.001$ \\
\hline & ECMO & $0(0.00 \%)$ & $1(2.86 \%)$ & $1(0.81 \%)$ & $<0.001$ \\
\hline \multirow[t]{2}{*}{ CT scores } & $1-4$ & $27(51.92 \%)$ & $20(40.00 \%)$ & $47(46.08 \%)$ & 0.227 \\
\hline & $5-7$ & $25(48.08 \%)$ & $30(60.00 \%)$ & 55 (53.92\%) & \\
\hline \multicolumn{6}{|l|}{ Disease progression } \\
\hline & Stableness/Hospitalization & $3(0.50 \%)$ & $3(2.73 \%)$ & $6(0.84 \%)$ & $<0.001$ \\
\hline & Improvement/Recover & $601(99.34 \%)$ & $101(91.82 \%)$ & $702(98.18 \%)$ & \\
\hline & Death & $1(0.17 \%)$ & $6(5.45 \%)$ & $7(0.98 \%)$ & \\
\hline $\begin{array}{l}\text { Length of hospital stay, d, } \\
\text { median (IQR) }\end{array}$ & & $20.0(14.0-25.0)$ & $20.0(15.0-28.0)$ & $20.00(14.00-26.00)$ & 0.243 \\
\hline ICU care & & $5(0.82 \%)$ & $24(20.00 \%)$ & 29 (93.55\%) & 0.007 \\
\hline \multicolumn{6}{|l|}{ Severity on admission } \\
\hline & Mild & 117 (19.24\%) & 20 (16.67\%) & 137 (18.82\%) & $<0.001$ \\
\hline & General & 365 (60.03\%) & 44 (36.67\%) & 409 (56.18\%) & \\
\hline & Severe & $122(20.07 \%)$ & 43 (35.83\%) & 165 (22.66\%) & \\
\hline & Critical & $4(0.66 \%)$ & 13 (10.83\%) & 17 (2.34\%) & \\
\hline \multicolumn{6}{|l|}{ Severity at worst } \\
\hline & Mild & 0 & 0 & 0 & $<0.001$ \\
\hline & General & 325 (53.5\%) & $18(15.0 \%)$ & 343 (47.18\%) & \\
\hline & Severe & 273 (45.0\%) & 76 (63.3\%) & 349 (48.01\%) & \\
\hline & Critical & $9(1.5 \%)$ & $26(21.7 \%)$ & 35 (4.81\%) & \\
\hline
\end{tabular}

TABLE 4 | The risk of elevated IL-6 for mortality of COVID-19.

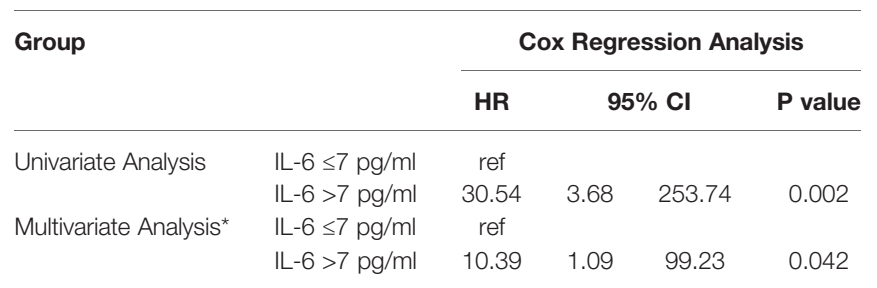

*Adjusted for age, history of cardiovascular disease, lymphocyte count, D-dimer, and lactate dehydrogenase.

\section{Mortality and IIIness Severity in Patients With Elevated IL-6 Levels}

The Kaplan-Meier curves and cumulative hazard function showed that patients with normal IL-6 levels had better survival ( $p<0.05$, Figures 1 and 2). Further, the ROC analysis showed that the elevated IL-6 group had the largest areas under the ROC curve (AUCs) of 0.870 and 0.778 for disease severity and CT scores, respectively (Figure 3).

\section{Curve Fitting and Trend Analyses for IL-6 Levels in COVID-19 Patients}

The peak value of Score 1 for all patients was 2.35 on day 23 (Figure 4A); however, while the peak value for the elevated IL-6 group was 2.46 on day 23 , the peak value in the normal IL-6 group was 2.24 on day 22 (Figure 4B). Nonetheless, the peak value of Score 1 was attained within the same period in both groups. The Score 2 -fitted curve for all patients presented a linear line that indicated a decline over time (Figure 4C). In the normal IL- 6 group, the fitted curve presented a more uniform declining linear line than that in the elevated IL-6 group (Figure 4D). For the overall score, all patients showed peak values on day 22 (4.60; Figure 4E). Patients in the elevated IL-6 group showed the peak score on day 24 (4.79; Figure 4E), whereas a fitted curve that presented a flattened, declining, linear line was seen for patients in the normal IL-6 group.

The curve-fitting analysis was used to evaluate the changes in IL-6 levels: there was a slight decrease on day 16 and an increase to the peak level on day 26 (Figure 5A). A similar trend and an obvious dynamic change in IL-6 levels were observed in the elevated IL-6 subgroup (Figure 5B).

\section{DISCUSSION}

Using data from a unique makeshift hospital for COVID-19 patients, this results from this retrospective study showed that higher serum levels of IL- 6 was an independent and reliable risk factor for COVID-19 patients and led to higher disease severity 
TABLE 5 | The risk of elevated IL-6 for disease severity of COVID- 19.

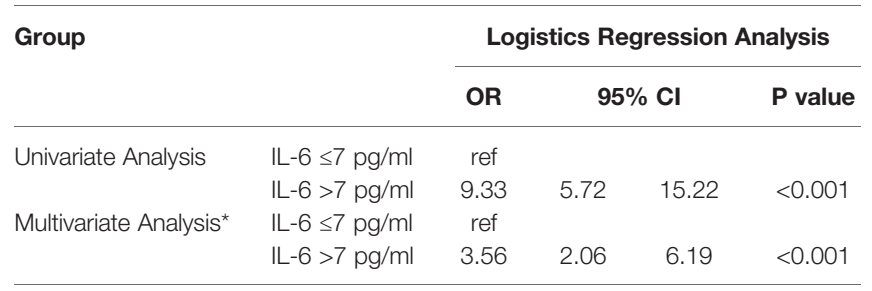

${ }^{*}$ Adjusted for age, history of cardiovascular disease, lymphocyte count, D-dimer, and lactate dehydrogenase.

and mortality. Moreover, the trend fluctuation occurred earlier than the negative changes in lung CT scores, which indicated a potential predictive ability for lung injury. These findings provide clinical evidence in support of recently published guidance statements by Aziz et al. (2020). Nevertheless, due to the programmatic, retrospective nature of this research, the inferences derived from the data require further validation from cohort studies and randomized controlled trials (RCTs).

IL-6, a chemokine secreted by T-cells and macrophages to stimulate the immune response, is an important biomarker of inflammation, and elevated IL-6 levels have been demonstrated in inflammatory states induced by several pathological conditions (Cai et al., 2020). In COVID-19 patients, overproduction of cytokines, such as IL-6, might activate coagulation pathways, with a resultant disruption of procoagulant-anticoagulant homeostasis, induction of disseminated intravascular coagulation, and multiorgan dysfunction or failure (Jose and Manuel, 2020). In this study, we found that COVID-19 patients with elevated IL-6 levels had a higher likelihood of experiencing renal, hepatic, and lung injuries (Table 2).

Previous clinical studies have shown that older age, male sex, cardiovascular diseases, lower lymphocyte counts, higher Ddimer levels, and increased lactate dehydrogenase levels are significant risk factors for illness severity or mortality in COVID-19 patients (Li et al., 2020; Wang et al., 2020). In this study, there were a larger proportion of patients with these in the elevated IL-6 group. Thus, patients with high IL-6 levels may have a worse prognosis. Research has demonstrated that a higher IL-6 level on admission can predict greater odds of complications and illness severity in COVID-19 patients (Liu et al., 2020); however, mortality was not evaluated. Although a few studies have indicated that increases in IL-6 levels are potentially associated with an increased likelihood of mortality, they lacked statistical power as residual confounders were not considered or only indirectly validated through lower mortality after using an interleukin-6 receptor antagonist for blockading cytokine release in severe COVID-19 patients (Alattar et al., 2020; Aziz et al., 2020; Zhang et al., 2020). A multivariate Cox regression model was developed in our study to adjust for confounders including older age, history of cardiovascular disease, lower lymphocyte count, higher D-dimer levels, and increased lactate dehydrogenase levels. These risk factors of mortality have been validated in previous research (Ji et al., 2020; Li et al., 2020; Zhou et al., 2020). Additionally, in our study, Kaplan-Meier and cumulative hazard analyses were conducted to verify the strong associations of IL-6 levels with disease severity and mortality. Thus, all analyses conducted in our study validated that elevated IL-6 levels are an independent risk factor for the severity and mortality of COVID-19.

Furthermore, we evaluated the potential predictive function of IL-6. COVID-19 is caused by severe acute respiratory syndrome coronavirus 2 (SARS-CoV-2) (Huang et al., 2020), which binds to the alveolar epithelial cells to subsequently activate innate and adaptive immune responses and induces the release of a large number of cytokines, including IL-6. Moreover, considering the inherent role of these proinflammatory factors, vascular permeability increases, followed by the influx of large amounts of fluid and a large number of blood cells into the alveoli, resulting in dyspnea or ARDS (Knudsen and Ochs, 2018; Leiva-Juarez et al., 2018). The flux in IL-6 levels is associated with the pathological processes that mediate lung injuries, which was demonstrated by the similarity in peak timings of IL-6 levels and CT scores (Figure 4) in this study. Interestingly, IL-6 levels showed a slight decrease prior
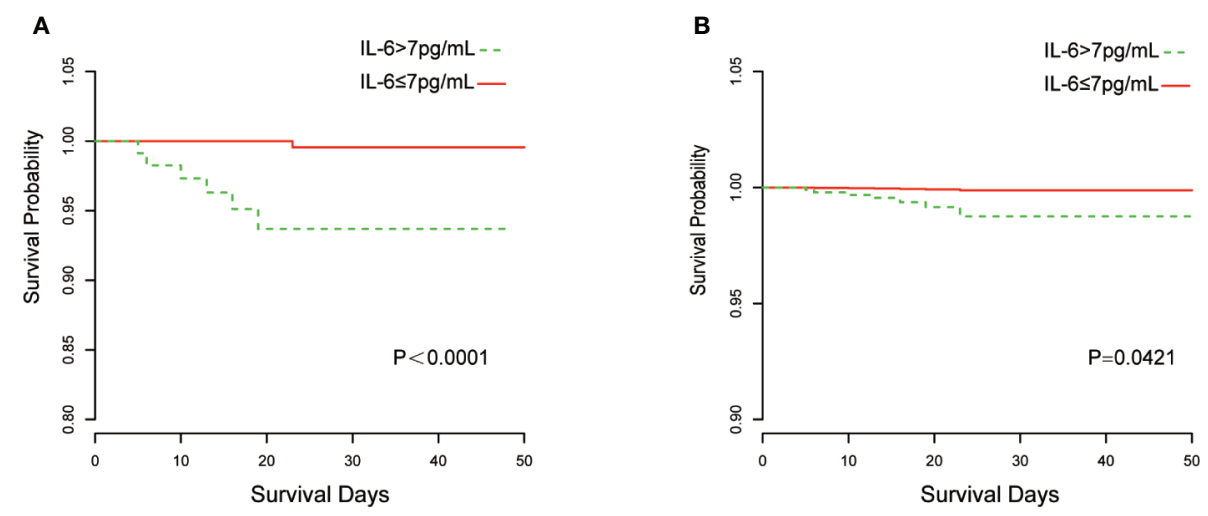

FIGURE 1 | Kaplan-Meier curves of IL-6 group stratification for survival. (A) Without adjustment; (B) Adjusted for age, the history of cardiovascular disease, lymphocyte count, D-dimer, and lactate dehydrogenase. 


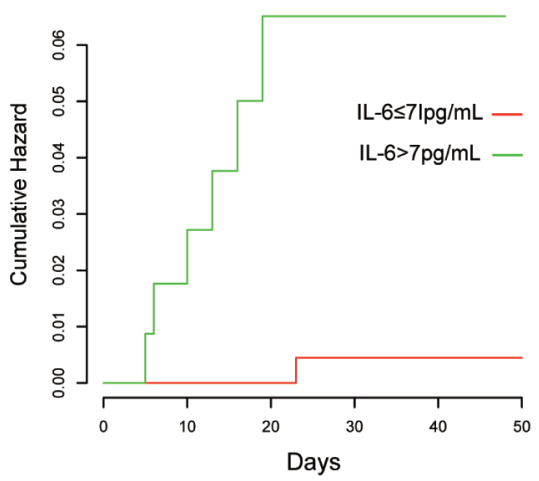

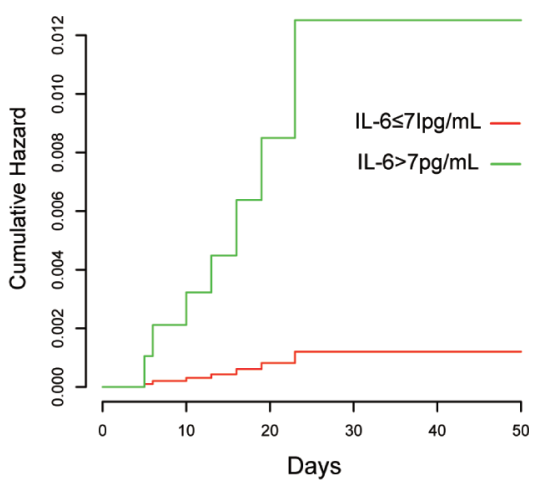

FIGURE 2 | Cumulative hazards of death are presented. (A) Without adjustment; (B) Adjusted for age, history of cardiovascular disease, lymphocyte count, Ddimer, and lactate dehydrogenase.

A

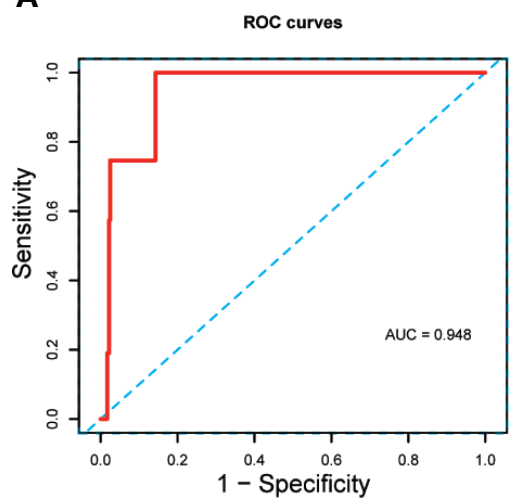

B

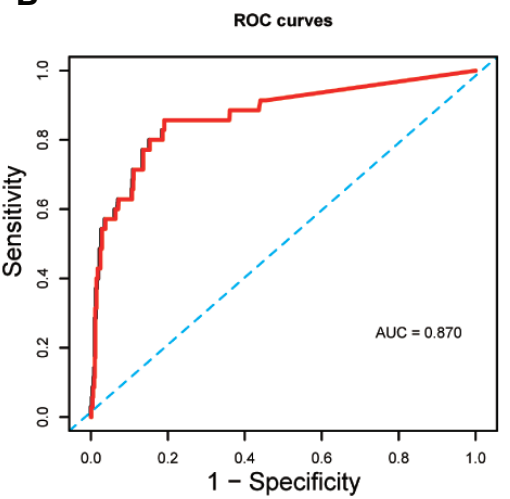

C

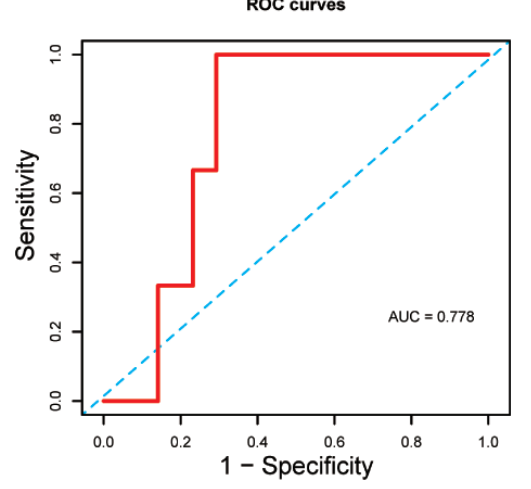

FIGURE 3 | (A) ROC curve assessed the predictive capability of elevated IL-6 for death; (B) ROC curve assessed the predictive capability of elevated IL-6 for disease severity; (C) ROC curve assessed the predictive capability of elevated IL-6 for CT scores.

to the increase in CT scores in the fitting curve. This may be a result of SARS-CoV-2 eliciting attenuated innate immunity, leading to delayed proinflammatory cytokine induction, similar to the reaction observed in patients infected with Middle East respiratory syndrome coronavirus (MERS-CoV) (Lau et al., 2013). Further, in the early phase of the cytokine storm, immune cells and cytokines may be clustered in the pulmonary lesions, resulting in a decrease in IL-6 levels in the peripheral blood. Consequently, we observed a mild decline occurred in IL-6 serum levels over the initial 10-20 days of the clinical course (Figure 4). In the management of SARS patients, dynamic changes in IL-6 levels have been shown to occur synchronously with the changes in radiographic scores (Chien et al., 2006). Moreover, a similar association between IL-6 levels and CT scores was observed in COVID-19 patients over a period of 20-30 days (Figure 5). The decrease in IL-6 levels occurred earlier than the increase in CT scores that reflected the progressive deterioration of lung lesions (Figure 5). The disparity in the two aforementioned scores was more obvious in the elevated IL- 6 group. Thus, the decreasing trend of IL-6 levels might be predictive of the imminent worsening of lung injuries. In addition, the good predictive capability of IL-6 for disease severity and prognosis in COVID-19 patients, assessed by the ROC curves (AUC > 0.75; Figure 3), provided further evidence regarding its predictive function.

Nonetheless, this study has several limitations. First, due to the programmatic and retrospective nature of this study, we could not measure serum IL-6 levels or assess CT images on the same day. Second, the size of the study sample was modest, and there was inadequate statistical power to detect whether there was a differential effect among the mild, severe, and critical COVID-19 cases. Third, as this was a retrospective study, data on all parameters were not available for all patients, and data regarding in-hospital medications may not have been fully recorded. Some patients were transferred from other hospitals and might not have received uniform treatment in the early stages of COVID-19. Fourth, testing for IL-6 levels was performed at different time intervals for 
A

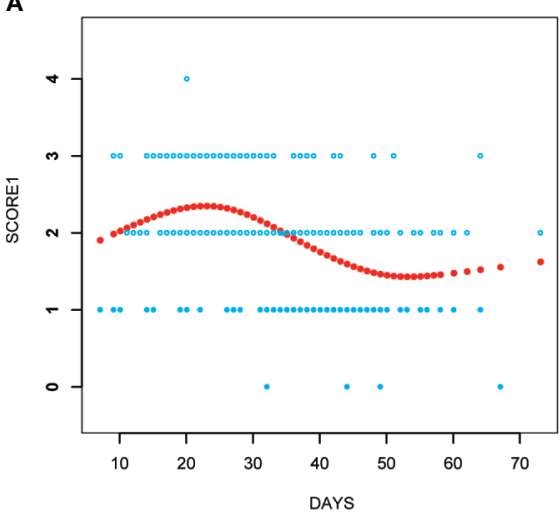

C

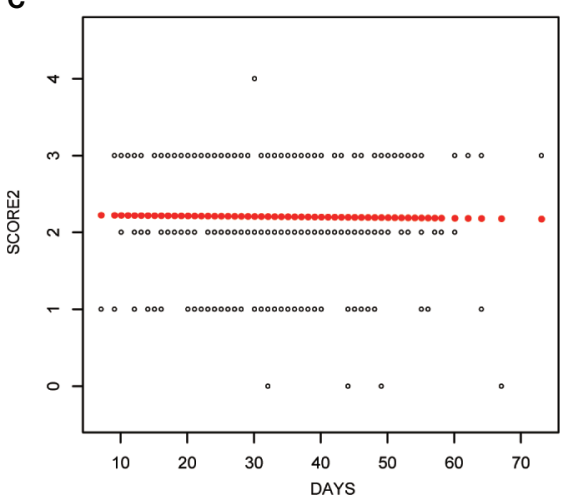

E

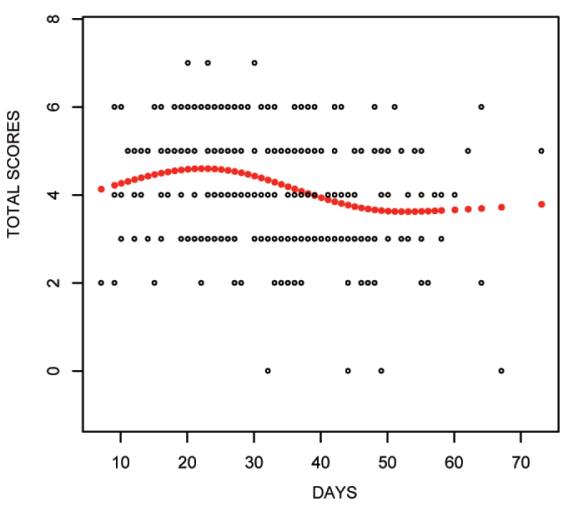

B

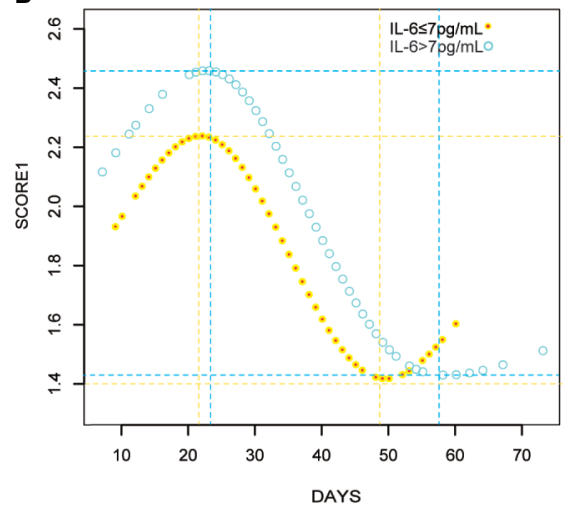

D

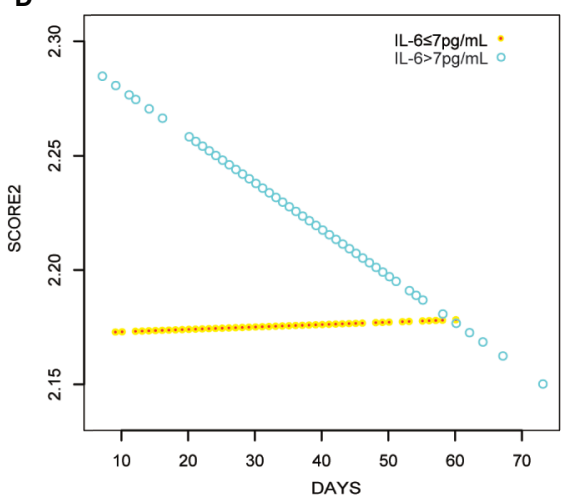

F

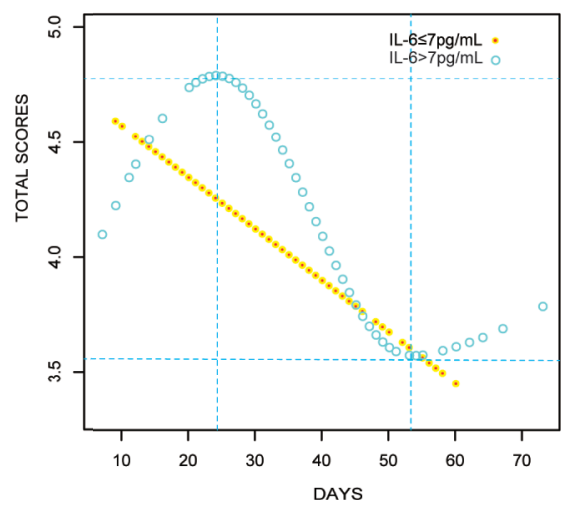

FIGURE 4 | Curve fitting analyses with CT scores for all COVID-19 patients (A, C, E); IL-6 elevated group (IL $>7$ pg/ml) versus IL-6 elevated group (IL $\leq 7$ pg/ml) (B, D, F).

each patient. Hence, diagnostic bias may have resulted from the increased number of tests performed in patients with high IL-6 levels. Fifth, we were unable to retrieve pre-hospital self-medication data from the in-hospital electronic records, considering the emergent circumstances of the COVID-19 pandemic. Sixth, median age was different between the elevated IL- 6 group and the normal IL- 6 group that may have caused bias in the analyses of different laboratory parameters, although we adjusted for age in our multivariate analysis. Finally, the use of some medications, such as immunomodulators used for suppressing an overactive cytokine response may have influenced the experimental results. There may have been unknown interactive effects between medications and IL-6.

\section{CONCLUSION}

The results from this study need to be interpreted considering other potential and residual confounders. It is likely that a higher IL-6 level is an independent risk factor for in-hospital severity and 

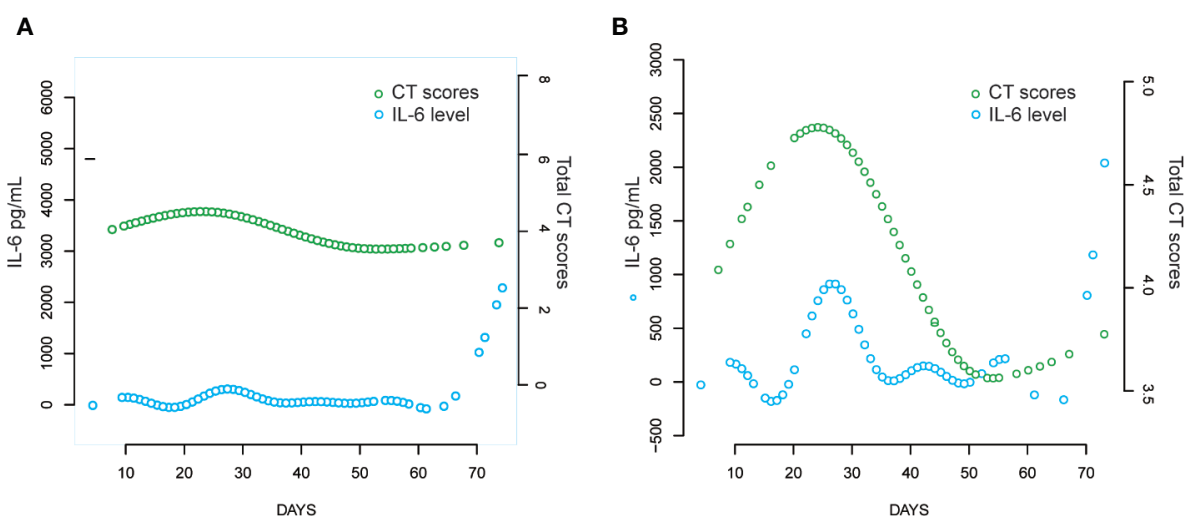

FIGURE 5 | Comparison of CT scores trend and IL-6 levels over time with curve fitting analyses. (A) All the COVID-19 patients; (B) Patients with elevated IL-6.

mortality in Chinese COVID-19 patients, and it might be a potential predictor of lung injury in these patients. The findings of this study provide novel research insights that may guide the early therapeutic intervention for COVID-19 patients, such as dietary interventions (Messina et al., 2020) and medication with IL-6 receptor blockers (Zhang et al., 2020). However, cohort studies, prospective studies, and RCTs are required to further validate the impact of these findings and the early therapeutic strategies.

\section{DATA AVAILABILITY STATEMENT}

The raw data supporting the conclusions of this article will be made available by the authors, without undue reservation.

\section{ETHICS STATEMENT}

The studies involving human participants were reviewed and approved by Research Ethics Committee of the Zhongnan Hospital of Wuhan University. Written informed consent for participation was not required for this study in accordance with the national legislation and the institutional requirements.

\section{AUTHOR CONTRIBUTIONS}

ZL, JL, DC, RG, and WZ designed the study, collected and analyzed the data, and wrote the manuscript. SC, YH, JH, WL,

\section{REFERENCES}

Ai, T., Yang, Z., Hou, H., Zhan, C., Chen, C., Lv, W., et al. (2020). Correlation of Chest CT and RT-PCR Testing in Coronavirus Disease 2019 (COVID-19) in China: A Report of 1014 Cases. Radiology, 200642. doi: 10.1148/radiol.2020200642 and ML collected and reviewed the clinical, laboratory, and radiological data. $\mathrm{ZL}, \mathrm{JL}$, and $\mathrm{DC}$ performed statistical analyses. RG and $\mathrm{WZ}$ reviewed, interpreted, and checked the clinical data. LG, XWa, and XWu wrote the manuscript and provided valuable suggestions for the study design and data analysis. LG, XWa, and XWu contributed equally, designed the project, edited the manuscript, and supervised the study. All authors contributed to the article and approved the submitted version.

\section{ACKNOWLEDGMENTS}

We would like to thank all patients and their families. As Wuhan was hit hard by the pandemic not long ago, its people made huge sacrifices to push back the virus. We also would like to thank the doctors, nurses, and other medical team members from different places across China. In our most difficult days, they chose to stay with us at the Leishenshan Hospital, offered a helping hand, and helped us make it through.

\section{SUPPLEMENTARY MATERIAL}

The Supplementary Material for this article can be found online at: https://www.frontiersin.org/articles/10.3389/fphar. 2020.01093/full\#supplementary-material

FIGURE S1 | Design schematic of the study. 
Cai, Q., Huang, D., Ou, P., Yu, H., Zhu, Z., Xia, Z., et al. (2020). COVID-19 in a designated infectious diseases hospital outside Hubei Province, China. Allergy 75, 1742-1752. doi: 10.1111/all.14309

Chen, G., Wu, D., Guo, W., Cao, Y., Huang, D., Wang, H., et al. (2020). Clinical and immunological features of severe and moderate coronavirus disease 2019. J. Clin. Invest. 130 (5), 2620-2629. doi: 10.1172/JCI137244

Chien, J. Y., Hsueh, P. R., Cheng, W. C., Yu, C. J., and Yang, P. C. (2006). Temporal changes in cytokine/chemokine profiles and pulmonary involvement in severe acute respiratory syndrome. Respirology 11 (6), 715722. doi: 10.1111/j.1440-1843.2006.00942.x

Huang, C., Wang, Y., Li, X., Ren, L., Zhao, J., Hu, Y., et al. (2020). Clinical features of patients infected with 2019 novel coronavirus in Wuhan, China. Lancet (London England) 395 (10223), 497-506. doi: 10.1016/s0140-6736(20)30183-5

Ji, D., Zhang, D., Xu, J., Chen, Z., Yang, T., Zhao, P., et al. (2020). Prediction for Progression Risk in Patients with COVID-19 Pneumonia: the CALL Score. Clin. Infect. Dis. doi: 10.1093/cid/ciaa414

Jose, R. J., and Manuel, A. (2020). COVID-19 cytokine storm: the interplay between inflammation and coagulation. Lancet Respir. Medicine. 8, e46-e47. doi: 10.1016/s2213-2600(20)30216-2

Knudsen, L., and Ochs, M. (2018). The micromechanics of lung alveoli: structure and function of surfactant and tissue components. Histochem. Cell Biol. 150 (6), 661-676. doi: 10.1007/s00418-018-1747-9

Lau, S. K. P., Lau, C. C. Y., Chan, K. H., Li, C. P. Y., Chen, H., Jin, D. Y., et al. (2013). Delayed induction of proinflammatory cytokines and suppression of innate antiviral response by the novel Middle East respiratory syndrome coronavirus: implications for pathogenesis and treatment. J. Gen. Virol 94 (Pt 12), 2679-2690. doi: 10.1099/vir.0.055533-0

Leiva-Juarez, M. M., Kolls, J. K., and Evans, S. E. (2018). Lung epithelial cells: therapeutically inducible effectors of antimicrobial defense. Mucosal Immunol. 11 (1), 21-34. doi: 10.1038/mi.2017.71

Li, X., Xu, S., Yu, M., Wang, K., Tao, Y., Zhou, Y., et al. (2020). Risk factors for severity and mortality in adult COVID-19 inpatients in Wuhan. J. Allergy Clin. Immunol. 146 (1), 110-118. doi: 10.1016/j.jaci.2020.04.006

Liu, F., Li, L., Xu, M., Wu, J., Luo, D., Zhu, Y., et al. (2020). Prognostic value of interleukin-6, C-reactive protein, and procalcitonin in patients with COVID19. J. Clin. Virol 127:104370. doi: 10.1016/j.jcv.2020.104370

Lyu, P., Liu, X., Zhang, R., Shi, L., and Gao, J. (2020). The performance of chest CT in evaluating the clinical severity of COVID-19 pneumonia: identifying critical cases based on CT characteristics. Invest. Radiol. doi: 10.1097/ rli.0000000000000689
Meduri, G. U., Kohler, G., Headley, S., Tolley, E., Stentz, F., and Postlethwaite, A (1995). Inflammatory cytokines in the BAL of patients with ARDS. Persistent elevation over time predicts poor outcome. Chest 108 (5), 1303-1314. doi: $10.1378 /$ chest.108.5.1303

Messina, G., Polito, R., Monda, V., Cipolloni, L., Di Nunno, N., Di Mizio, G., et al. (2020). Functional Role of Dietary Intervention to Improve the Outcome of COVID-19: A Hypothesis of Work. Int. J. Mol. Sci. 21 (9), 3104-3118. doi: 10.3390/ijms21093104

Ranieri, V. M., Rubenfeld, G. D., Thompson, B. T., Ferguson, N. D., Caldwell, E., Fan, E., et al. (2012). Acute respiratory distress syndrome: the Berlin Definition. JAMA 307 (23), 2526-2533. doi: 10.1001/jama.2012.5669

Wang, K., Zhang, Z., Yu, M., Tao, Y., and Xie, M. (2020). 15-day mortality and associated risk factors for hospitalized patients with COVID-19 in Wuhan, China: an ambispective observational cohort study. Intensive Care Med. 46 (7), 1472-1474. doi: 10.1007/s00134-020-06047-w

Wong, K. T., Antonio, G. E., Hui, D. S., Ho, C., Chan, P. N., Ng, W. H., et al. (2004). Severe acute respiratory syndrome: thin-section computed tomography features, temporal changes, and clinicoradiologic correlation during the convalescent period. J. Comput. Assist Tomogr 28 (6), 790-795. doi: 10.1097/ 00004728-200411000-00010

Zhang, C., Wu, Z., Li, J. W., Zhao, H., and Wang, G. Q. (2020). The cytokine release syndrome (CRS) of severe COVID-19 and Interleukin-6 receptor (IL6R) antagonist Tocilizumab may be the key to reduce the mortality. Int. J. Antimicrob. Agents 105954. doi: 10.1016/j.ijantimicag.2020.105954

Zhou, F., Yu, T., Du, R., Fan, G., Liu, Y., Liu, Z., et al. (2020). Clinical course and risk factors for mortality of adult inpatients with COVID-19 in Wuhan, China: a retrospective cohort study. Lancet 395 (10229), 1054-1062. doi: 10.1016/ S0140-6736(20)30566-3

Conflict of Interest: The authors declare that the research was conducted in the absence of any commercial or financial relationships that could be construed as a potential conflict of interest.

Copyright (c) $2020 \mathrm{Liu}, \mathrm{Li}$, Chen, Gao, Zeng, Chen, Huang, Huang, Long, Li, Guo, Wang and Wu. This is an open-access article distributed under the terms of the Creative Commons Attribution License (CC BY). The use, distribution or reproduction in other forums is permitted, provided the original author(s) and the copyright owner(s) are credited and that the original publication in this journal is cited, in accordance with accepted academic practice. No use, distribution or reproduction is permitted which does not comply with these terms. 